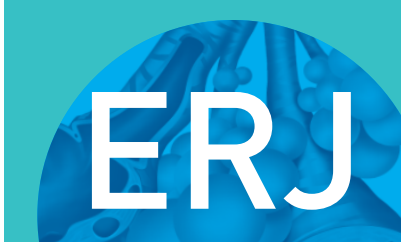

open research
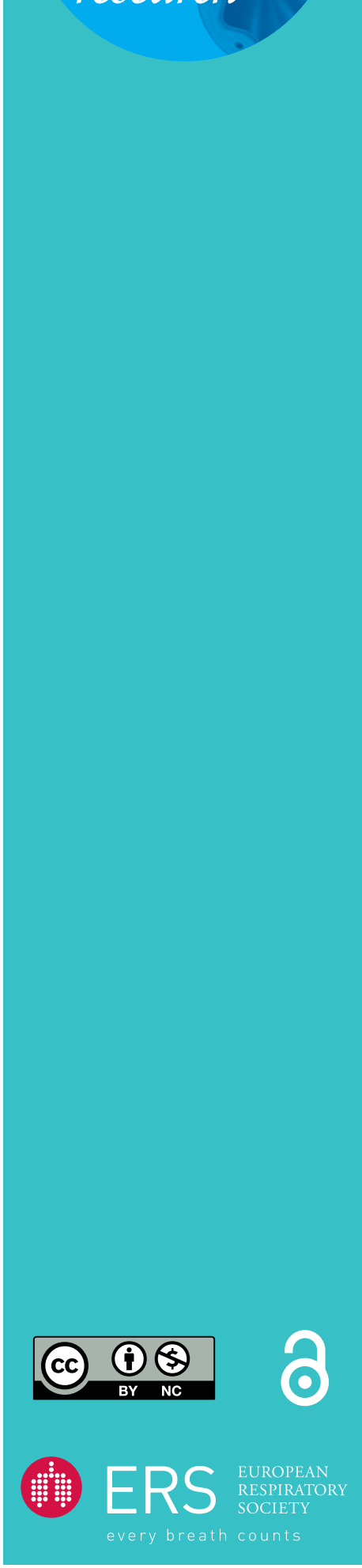

\section{Use of adjunct cardiovascular therapy in patients hospitalised for acute exacerbations of COPD}

\author{
To the Editor:
}

Acute exacerbation of chronic obstructive pulmonary disease (AECOPD) is one of the most frequent diagnoses in patients presenting with acute dyspnoea or respiratory failure. According to the Global Initiative for Chronic Obstructive Lung Disease (GOLD) document, it is defined clinically as acute worsening of respiratory symptoms that result in additional therapies, bronchodilators, corticosteroids and antibiotics being the cornerstone of acute management [1].

However, comorbidities in COPD, including cardiac disease, contribute significantly to the heterogeneity of single acute episodes in real-life practice [2]. This might determine changes in pharmacological treatment with adjunct therapy such as diuretics [3]. Therefore, it is sometimes difficult to discriminate between true exacerbations of COPD and acute worsening of a patient's condition due to associated comorbidities [4]. Furthermore, the lung-heart interplay is complex and AECOPD has been also correlated with alteration of haemodynamics [5]. Nevertheless, data are still scarce on the actual use of therapies such as diuretics during hospitalisation for AECOPD. Therefore, we were interested in evaluating how patients admitted to the hospital with a clinical diagnosis of AECOPD were managed at admission and we analysed the therapeutic approach at onset of AECOPD in hospitalised patients, aiming to assess the adjunct use of diuretic therapy.

This study was a retrospective, observational, single-centre study based on hospital records. The study population included all patients admitted to the unit of respiratory disease at our university hospital with a clinical diagnosis of AECOPD and respiratory failure from September 1, 2014, to September 30, 2015. Cases were identified retrospectively by evaluating International Classification of Diseases (Ninth Revision) diagnosis-related group codes then hospital records were reviewed for each patient to confirm inclusion. Patients with a previous clinical and/or functional diagnosis of COPD presenting to the hospital for respiratory failure (i.e. ratio between arterial blood partial oxygen pressure and inspired oxygen fraction $<300$ and an arterial blood partial carbon dioxide pressure $>45 \mathrm{mmHg}$ ) were considered for inclusion. Exclusion criteria were incomplete data on medications used, absence of a previous clinical diagnosis of COPD and respiratory failure due to a different cause (e.g. pneumothorax, acute cardiac disease, pulmonary embolism or acute heart failure, excluded both through the clinical assessment achieved with bedside cardiac transthoracic echocardiography and the results of serological evaluation of cardiac enzymes).

The following data were collected for each patient at admission: demographics and past medical history, Anthonisen criteria for lung infection [6], clinical signs of fluid overload (i.e. dyspnoea plus at least one of pulmonary rales, pulmonary oedema, peripheral oedema, orthopnoea or ascites [7]), signs of fluid overload (upper zone flow redistribution, lung interstitial oedema, alveolar pulmonary oedema or bilateral pleural effusion [8]) on the chest radiograph and/or computed tomography scan, blood gases, and laboratory tests. Short-term outcomes and vital status were recorded for completeness but were outside the scope of the current analysis. The study protocol was presented to the institutional ethics committee, which authorised data extraction. Informed consent was not required.

@ERSpublications

In real-life practice, acute exacerbation of COPD is often treated as a cardiopulmonary syndrome http://ow.ly/uAnk30luMYz

Cite this article as: Roversi $\mathrm{S}$, Tonelli $\mathrm{R}$, Beghè $\mathrm{B}$, et al. Use of adjunct cardiovascular therapy in patients hospitalised for acute exacerbations of COPD. ERJ Open Res 2018; 4: 00087-2018 [https:// doi.org/10.1183/23120541.00087-2018].

Copyright @ERS 2018. This article is open access and distributed under the terms of the Creative Commons Attribution NonCommercial Licence 4.0. 
Summary descriptive statistics were used to describe the study population and a multivariable logistic regression model was used to evaluate clinical features related to the use of diuretics. Independent variables were age, sex, number of comorbidities, Anthonisen criteria (present/absent), and radiological and clinical signs of overload (present/absent). All analyses were performed by means of R 3.3.2 statistical software (The R Foundation for Statistical Computing, Vienna, Austria) at the 95\% confidence level.

The study population included 122 elderly patients (average age 75.2 years, sD 9 years) with AECOPD, of whom 14 had two hospitalisations within the study period and four had three or more hospitalisations. The total number of recorded episodes was 146.

$57 \%$ of the patients were male and $91 \%$ were current or former smokers. All had a confirmed or reported diagnosis of COPD, of whom $8 \%$ were mild-moderate and $49 \%$ severe. Comorbidities were very frequent. Cardiac diseases such as heart failure, ischaemic heart disease and arrhythmias were common, and were reported in $71(58.2 \%)$ patients, often with the same patients presenting more than one diagnosis (i.e. 24 patients reported ischaemic heart disease, 44 reported heart failure, and 42 reported atrial fibrillation and/or other arrhythmias). Similarly, hypertension, diabetes, gastroenteric diseases and neoplasms were frequent in the study sample $(55.2 \%, 26.7 \%, 32 \%$ and $30.2 \%$, respectively). Associated lung disorders were not as common, with $8 \%$ reporting pulmonary hypertension and $6.5 \%$ reporting previous pulmonary embolism.

At admission, all patients presented significant worsening dyspnoea but clinical signs of fluid overload were present only in a minority of cases (28\%). Conversely, radiological signs were reported in $49 \%$, the majority being interstitial oedema and bilateral pleural effusion. Symptoms suggesting lung infections were present in $40 \%$. Cardiac biomarkers such as brain natriuretic peptide (BNP) and cardiac troponin $\mathrm{T}$ (cTnT) were available only in six and 42 patients, respectively, and proved, on average, to be raised (BNP $570 \mathrm{pg} \cdot \mathrm{mL}^{-1}$ and $\mathrm{cTnT} 0.14 \mathrm{ng} \cdot \mathrm{mL}^{-1}$ ). The diagnosis of associated heart failure was, however, confirmed and reported in hospital in only $9 \%$ of subjects.

Patients were mostly hypoxic and hypercapnic (mean arterial oxygen tension $64 \mathrm{mmHg}$ and carbon dioxide tension $54 \mathrm{mmHg}$ ) without severe or life-threatening acidosis. $25 \%$ of admitted patients required noninvasive ventilation, followed by endotracheal intubation in three of them.

Not surprisingly, the majority of patients received bronchodilators, corticosteroids and antibiotics but, quite interestingly, the vast majority of them (81\%) received adjunct loop diuretics, mostly furosemide (mean dosage $48 \mathrm{mg}$, sD $59 \mathrm{mg}$ ). Cardiac medications, proton-pump inhibitors and antidepressants were prescribed frequently.

Table 1 describes the frequency of drugs used as the first approach to manage AECOPD and reports factors associated with the use of diuretics assessed by means of multivariable logistic regression performed on the sample of 114 patients who had complete data for the variables of interest.

Our study is a description of current medical practice in patients hospitalised with respiratory failure and AECOPD episodes, defined clinically as in the GOLD document, and provides data on drug management during AECOPD episodes in real-life practice.

Overall, the population in study was old and multimorbid, which is in wide agreement with recent literature [9]. Similarly, the clinical presentation was not surprising: the AECOPD patients presented with alterations in gas exchange, half of them had pulmonary crackles, the majority presented wheezing and there were signs of congestion in $\sim 20 \%$ of cases $[10,11]$.

However, acute management with drug strategies at bedside offer some interesting insights. As expected, bronchodilators, corticosteroids and antibiotics were widely used in our population. However, in $17 \%$ of the episodes, patients were not started on bronchodilators; nonetheless, half of them received systemic corticosteroids. Antibiotics were used in $58 \%$ of the cases, which is lower than reported in other observational data on COPD $[12,13]$. On the contrary, cardiac drugs and diuretics were used more frequently than expected (e.g. $\beta$-blockers in $35 \%$ ). The factors that were significantly related to the use of diuretics were age, clinical signs of fluid overload and Charlson comorbidity index, with clinical signs of fluid overload having five-fold higher odds. Conversely, neither Anthonisen criteria nor radiological signs of overload were correlated.

Data from the literature generally report lower use of diuretics during AECOPD: loop diuretics were used in $35 \%$ of $>84000$ episodes across the USA, with only $13 \%$ of patients taking $\beta$-blockers [14]. In contrast, a smaller study specifically investigating heart failure in patients with AECOPD reported that diuretics were used in $31 \%$ of cases overall but the rate increased to $76 \%$ when patients with known concomitant COPD and heart failure were considered [15].

Taken together, the present data indicate that the clinical diagnosis of AECOPD, as performed in everyday practice, is rather unspecific, and a variety of clinical scenarios may be included under the umbrella of the 


\begin{tabular}{|c|c|c|}
\hline Drug & \multicolumn{2}{|c|}{ Patients } \\
\hline Antibiotics (intravenous) & \multicolumn{2}{|c|}{86 (77), $58.9 \%$} \\
\hline Nebulised bronchodilators & \multicolumn{2}{|c|}{$99,67.8 \%$} \\
\hline LABA & \multicolumn{2}{|c|}{$20,13.7 \%$} \\
\hline LAMA & \multicolumn{2}{|c|}{$16,10.9 \%$} \\
\hline ICS & \multicolumn{2}{|c|}{$19,13.0 \%$} \\
\hline Corticosteroids (intravenous) & \multicolumn{2}{|c|}{104 (96), $71.2 \%$} \\
\hline Diuretics (intravenous) & \multicolumn{2}{|c|}{118 (59), $80.8 \%$} \\
\hline$\beta$-blockers & \multicolumn{2}{|c|}{$52,35.6 \%$} \\
\hline Antihypertensive drugs & \multicolumn{2}{|c|}{$77,52.7 \%$} \\
\hline Antiplatelet drugs & \multicolumn{2}{|c|}{$57,39.0 \%$} \\
\hline Anticoagulants & \multicolumn{2}{|c|}{$42,28.7 \%$} \\
\hline Statins & \multicolumn{2}{|c|}{$50,34.2 \%$} \\
\hline Mineral receptor antagonists & \multicolumn{2}{|c|}{$43,29.4 \%$} \\
\hline Digitalis & \multicolumn{2}{|c|}{$9,6.0 \%$} \\
\hline Antidiabetic agents (insulin/oral hypoglycaemic drugs) & \multicolumn{2}{|c|}{29 (17/15), 19.8\% } \\
\hline Proton pump inhibitors & \multicolumn{2}{|c|}{$105,71.9 \%$} \\
\hline Antidepressants & \multicolumn{2}{|c|}{$71,48.6 \%$} \\
\hline Variable & OR $(95 \% \mathrm{CI})$ & p-value \\
\hline Age & $1.10(1.03-1.17)$ & 0.0032 \\
\hline Female sex versus male & $0.94(0.30-2.92)$ & 0.9108 \\
\hline Charlson comorbidity index & $1.86(1.29-2.69)$ & 0.0009 \\
\hline Anthonisen criteria present versus absent & $0.79(0.45-1.39)$ & 0.4067 \\
\hline Radiologic signs of overload present versus absent & $0.57(0.16-2.07)$ & 0.3944 \\
\hline Clinical signs of overload present versus absent & $5.19(1.05-25.62)$ & 0.0434 \\
\hline
\end{tabular}

same diagnosis. For example, the low rate of clinical signs of infection (and therefore, the relatively low use of antibiotics) is rather unexpected, since infection is generally indicated as the most common cause of AECOPD [2]. Similarly, we expected that diuretics would have been used commonly but we did not expect to find more patients being treated with diuretics than with nebulised bronchodilators. As our population comprised elderly, multimorbid patients, we hypothesised that the acute exacerbations of COPD were probably associated with alteration of the heart loading conditions and, thus, with impairment of heart function. Possibly, the majority of patients presented clinical features evocative of heart failure, and thus were deemed to benefit from adjunctive treatment. Furthermore, it is known that heart failure is frequent among patients with COPD and that AECOPD is a well-known cause of worsening heart failure [9-15]. Thus, some of our patients hospitalised for AECOPD could have a clinical presentation evocative of associated heart failure, albeit without a formal diagnosis of overt cardiac disease. Whether heart failure may be a cause of AECOPD as common as lung infection is unknown but our data suggest that it is a field worth exploring.

Notwithstanding, our findings should be interpreted with caution due to the retrospective nature of the study. In particular, the diagnosis of COPD was not confirmed by spirometry in all patients, data were missing for ECG and echocardiography, and the small number of patients limits analysis and impairs causal correlation. More specifically, the study data do not allow a throughout analysis of the aetiology underling the acute exacerbation of respiratory symptoms in patients with COPD and therefore, our findings should be regarded mainly as hypothesis generating. However, it is reasonable to deduce that the attending physician felt the need for adjunctive diuretics beyond standard respiratory treatment in the majority of patients hospitalised with a diagnosis of exacerbation of COPD, which is a rather novel finding in AECOPD literature.

In conclusion, we could argue that in real-life practice, acute exacerbation of COPD is often treated as a cardiopulmonary syndrome. Although speculative, our findings warrant further evaluation, with appropriate prospective studies to explore this topic. 
Sara Roversi ${ }^{1}$, Roberto Tonelli ${ }^{1}$, Bianca Beghè ${ }^{1}$, Federico Banchelli ${ }^{2}$, Roberto D'Amico ${ }^{2}$, Mario Malerba ${ }^{3}$, Leonardo M. Fabbri $\oplus^{4,5}$ and Enrico M. Clini $\oplus^{1}$

${ }^{1}$ Dept of Medical and Surgical Sciences, University of Modena and Reggio Emilia and Respiratory Diseases Unit, Modena, Italy. ${ }^{2}$ Statistic Unit, Dept of Diagnostics, Clinical and Public Health Medicine, University of Modena and Reggio Emilia, Modena, Italy. ${ }^{3}$ San Andrea Hospital-ASL Vercelli, Pneumology Unit, Dept of Translational Medicine, University of Piemonte Orientale, Novara, Italy. ${ }^{4}$ Dept of Medical Sciences, University of Ferrara, Ferrara, Italy. ${ }^{5}$ Sahlgresnska University Hospital, Gothenburg, Sweden.

Correspondence: Enrico M. Clini, Dept of Medical and Surgical Sciences, University of Modena and Reggio Emilia, Largo del Pozzo 71, Modena 41124, Italy. E-mail: enrico.clini@unimore.it

Received: June 122018 | Accepted after revision: Aug 172018

Conflict of interest: None declared.

\section{References}

1 Vogelmeier CF, Criner GJ, Martinez FJ, et al. Global Strategy for the Diagnosis, Management, and Prevention of Chronic Obstructive Lung Disease 2017 Report: GOLD Executive Summary. Eur Respir J 2017; 49: 1700214.

2 Wedzicha JA, Seemungal T. COPD exacerbations: defining their cause and prevention. Lancet 2007; 370: 786-796.

3 Abroug F, Ouanes-Besbes L, Nciri N, et al. Association of left-heart dysfunction with severe exacerbation of chronic obstructive pulmonary disease. Am J Respir Crit Care Med 2006; 174: 990-996.

4 Roca M, Verduri A, Corbetta L, et al. Mechanisms of acute exacerbation of respiratory symptoms in chronic obstructive pulmonary disease. Eur J Clin Invest 2013; 43: 510-521.

5 MacDonald MI, Shafuddin E, King PT, et al. Cardiac dysfunction during exacerbations of chronic obstructive pulmonary disease. Lancet Respir Med 2016; 4: 138-148.

6 Anthonisen NR, Manfreda J, Warren CP, et al. Antibiotic therapy in exacerbations of chronic obstructive pulmonary disease. Ann Intern Med 1987; 106: 196-204.

7 Felker GM, Lee KL, Bull, DA, et al. Diuretic strategies in patients with acute decompensated heart failure. $N$ Engl J Med 2011; 364: 797-805.

8 Fonseca $\mathrm{C}$, Mota $\mathrm{T}$, Morais $\mathrm{H}$, et al. The value of the electrocardiogram and chest X-ray for confirming or refuting a suspected diagnosis of heart failure in the community. Eur J Heart Fail 2004; 6: 807-812.

9 Almagro P, Cabrera FJ, Diez J, et al. Comorbidities and short-term prognosis in patients hospitalized for acute exacerbation of COPD: the EPOC en Servicios de medicina interna (ESMI) study. Chest 2012; 142: 1126-1133.

10 Roche N, Zureik M, Soussan D, et al. Predictors of outcomes in COPD exacerbation cases presenting to the emergency department. Eur Respir J 2008; 32: 953-996.

11 Wedzicha JA, Singh R, Mackay AJ. Acute COPD exacerbations. Clin Chest Med 2014; 35: 157-163.

12 Lindenauer PK, Pekow PS, Lahti MC, et al. Association of corticosteroid dose and route of administration with risk of treatment failure in acute exacerbation of chronic obstructive pulmonary disease. JAMA 2010; 303: 2359-2367.

13 López-Campos JL, Hartl S, Pozo-Rodriguez F, et al. Variability of hospital resources for acute care of COPD patients: the European COPD Audit. Eur Respir J 2014; 43: 754-762.

14 Rothberg MB, Pekow PS, Lahti M, et al. Antibiotic therapy and treatment failure in patients hospitalized for acute exacerbations of chronic obstructive pulmonary disease. JAMA 2010; 303: 2035-2042.

15 Minora T, Marino P. Peptidi natriuretici e broncopneumopatia cronica ostruttiva nel Dipartimento di Emergenza [B-type natriuretic peptide and COPD in the Emergency Department]. Ital J Med 2010; 4: 247-253. 